\title{
Morfologia polínica de espécies de Chrysophyllum L. (Sapotaceae) do Estado da Bahia, Brasil
}

\author{
Pollen morphology of species of Chrysophyllum L. (Sapotaceae) from Bahia State, Brazil
}

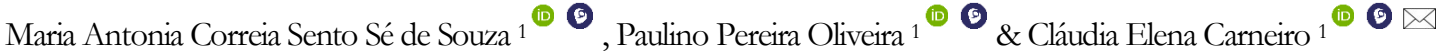

\section{Universidade Estadual de Feira de Santana, Departamento de Ciências Biológicas, Laboratório de Micromor- fologia Vegetal, Feira de Santana, Bahia, Brasil}

Palavras-chave:

Flora da Bahia. Grãos de pólen. Palinologia.

\section{Keywords:}

Flora of Bahia. Pollen grains. Palynology.

Recebido em: 11/04/2021

Aceito em: 21/07/2021

Editor responsável: Jaílson S. de Novais (UFSB)

eISSN: 2595-6752

\section{(c) (i)}

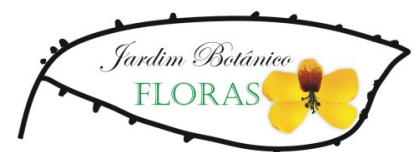

\begin{abstract}
Resumo
A morfologia polínica de nove espécies de Chrysophyllum (Sapotaceae) foi descrita sob a microscopia de luz. Os grãos de pólen foram coletados de amostras herborizadas, acetolisados, montados entre lâminas e lamínulas, analisados quantitativa e qualitativamente, descritos e fotomicrografados. As espécies estudadas de Chrysophyllum caracterizaram-se por apresentarem grãos de pólen mônades, tamanho variando de pequenos à médios, isopolares, com forma subprolata a prolata, amb subtriangular, subcircular e subquadrangular, 3-4-colporados, exina psilada, microrreticulada, levemente estriada. As espécies de Chrysophyllum estudadas apresentaram grãos de pólen com pequenas variações morfológicas, especialmente quanto ao número apertural e à ornamentação da exina. Assim, por se tratar do primeiro estudo na Bahia, abordando a morfologia polínica do gênero, poderá subsidiar futuros trabalhos, tanto local como regional, nas diversas áreas da Palinologia.
\end{abstract}

\section{Abstract}

The pollen morphology of nine species of Chrysophyllum (Sapotaceae) was described under light microscopy. Pollen grains were collected from herborized samples. Then, they were acetolysed, placed on slides, analyzed quantitatively and qualitatively, described and photomicrographed. The species of Chrysophyllum were characterized by presenting monad pollen grains, size ranging from small to medium, isopolar, subprolate to prolate shaped, subtriangular, subcircular and subquadrangular, 3-4 colporate, exine psilate, microreticulate and slightly striate. The Chrysophyllum species presented pollen grains with small morphological variations, especially regarding the aperture number and exine ornamentation. Thus, as the present study was one of the first made in Babia about the pollen morphology of the genus, it may support future work, both local and regional, in the various areas of Palynology.

\section{Introdução}

A familia Sapotaceae, inclusa na ordem Ericales (APG IV, 2016), tem distribuição predominantemente pantropical, constituída por 53 gêneros e 1.250 espécies, sendo encontrada principalmente em regiões tropicais e subtropicais da Ásia e América do Sul (Pennington, 1990; Swenson; Anderberg, 2005; Judd et al., 2009). Suas espécies estão distribuídas em três subfamílias: Sarcospematoideae, Sapotoideae e Chrysophylloideae (Swenson; Anderberg, 2005; Faria et al., 
2017), ocorrendo preferencialmente em florestas úmidas, abaixo de $1.000 \mathrm{~m}$ de altitude, e seu principal centro de diversidade é a América tropical (Pennington, 2004; APG IV, 2016).

No Brasil, a família Sapotaceae está representada por 13 gêneros e 240 espécies, estimando-se para a Bahia a ocorrência de aproximadamente 80 espécies distribuídas em 12 gêneros (Alves-Araújo et al., 2020). Acredita-se que ainda existam espécies não descritas para o Estado, assim como novas ocorrências, principalmente em formações florestais do sul baiano.

Taxonomicamente, os gêneros desta família apresentam problemas na delimitação de suas espécies, dificultando a identificação correta de seus táxons, principalmente devido à carência de dados morfológicos que possam sustentar as relações existentes entre eles.

O gênero Chrysophyllum L. é o segundo maior gênero de Sapotaceae em número de espécies, abrangendo em torno de 31 espécies na região neotropical (Pennington, 1990). A maioria das espécies de Chrysophyllum ocorre na América do Sul, principalmente em regiões de floresta úmida de baixada. No Brasil, os centros de dispersão do gênero são a Amazônia, principalmente, e a região costeira, com a ocorrência mais frequente de espécies nos estados da Bahia, Espírito Santo, Rio de Janeiro e São Paulo (Pennington, 1991).

Em se tratando de estudos palinológicos, o trabalho mais completo sobre Sapotaceae foi realizado por Harley (1991) para espécies neotropicais, embora ainda insuficiente para a caracterização polínica da família. Dados palinológicos para as espécies de Chrysophyllum são encontrados em catálogos e/ou floras polínicas para poucas espécies (Roubik; Moreno, 1991; Perveen; Qaiser, 2002; Melhem et al., 2003; Melo et al., 2017). Estudos palinológicos que tratem exclusivamente do gênero são inexistentes, justificando assim estudos mais específicos para o referido grupo.

O presente estudo foi realizado com o objetivo de caracterizar a morfologia polínica de espécies de Chrysophyllum que ocorrem no estado da Bahia, visando identificar caracteres informativos que possam colaborar para o melhor conhecimento palinológico sobre o gênero, assim como auxiliar no entendimento da taxonomia do grupo.

\section{Material e Métodos}

O material polinífero de Chrysophyllum foi coletado em exsicatas e/ou duplicatas depositadas no Herbário da Universidade Estadual de Feira de Santana (HUEFS), obtendo-se sempre que possível, botões florais de, no mínimo, três espécimes por espécie. Devido à falta de material polinífero em espécimes coletados na Bahia, amostras de outros estados foram examinados. Foram estudadas nove espécies: Chrysophyllum arenarium Allemão, C. flexuosum Mart., C. gonocarpum (Mart. \& Eichler ex Miq.) Engl., C. inornatum Mart., C. januariense Eichler, C. marginatum (Hook. \& Arn.) Radlk., C. rufum Mart., C. splendens Spreng., C. viride Mart. \& Eichler.

$\mathrm{O}$ processamento químico do material polinífero seguiu o método de acetólise segundo Erdtman (1960), e os grãos de pólen foram montados entre lâminas e lamínulas com gelatina glicerinada e seladas com parafina.

Os grãos de pólen foram analisados qualitativa e quantitativamente sob microscópio de luz. Para os parâmetros morfométricos (diâmetros equatorial e polar) foram tomadas 25 medidas dos grãos de pólen ao acaso, em aumento de 400x. Demais parâmetros como espessura da exina (sexina e nexina) foram medidos em dez grãos de pólen, na região polar (em vista equatorial), em aumento de $1.000 \times$. Os dados quantitativos foram submetidos à análise estatística, calculando-se a média aritmética $(\widehat{x})$, o desvio padrão da média $(S \bar{x})$, o desvio padrão da amostra (s), o coeficiente de variabilidade (CV) e o intervalo de confiança a $95 \%$ (IC), para as mensurações dos parâmetros polínicos com tamanho amostral igual a 25 . Para as medidas com tamanho amostral igual a dez foi calculada apenas a média aritmética. Para a espécie C. flexuosum não foi possível estabelecer o amb. por não ter sido encontrado nenhum grão de pólen em vista polar. Também não foi possível obter medidas do diâmetro equatorial, na vista polar, para todas as espécies, devido à tendência dos grãos de pólen caírem preferencialmente em vista equatorial, nas lâminas.

O material polínico foi ilustrado através de fotomicrografias. A terminologia adotada seguiu a nomenclatura de Punt et al. (2007). As lâminas foram depositadas na Palinoteca do Laboratório de Micromorfologia Vegetal (PUEFS) da Universidade Estadual de Feira de Santana.

\section{Resultados}

As espécies de Chrysophyllum estudadas caracterizaram-se por apresentarem grãos de pólen em mônades, com tamanho variando de pequenos a médios, isopolares, forma subprolata a prolata, amb subtriangular, subcircular e subquadrangular, 3-4-colporados, ectoabertura variando de curtas a longas, endoaberturalalongadas curtas, levemente costadas, com presença de fastígio em algumas espécies, exina psilada, microrreticulada, levemente estriada. Os dados da morfometria polínica estão apresentados na Tabela 1.

\section{Chrysophyllum arenarium Allemão (Figura 1a)}

Grãos de pólen em mônades, pequenos, isopolares, prolatos, amb. subcircular a subtriangular, 3-(4)-colporados, planaperturados, ectoaberturas estreitas, endoaberturas lalongadas curtas e levemente costadas. Exina psilada.

Material estudado - BRASIL, Ceará: Barbalha, 26/III/2008, Lorenæi 6475 (HUEFS).

Chrysophyllum flexuosum Mart. (Figura 1b)

Grãos de pólen em mônades, médios, isopolares, subprolatos, (3)-4 -colporados, planaperturados, endoaberturas lalongadas, curtas e levemente costadas. Exina psilada.

Material examinado - BRASIL, São Paulo: Pariquera-Açu, 07/ I/1999, Sztutman 135 (HUEFS). Bahia: Mata de São João, 11/ VI/2012, Miranda et al. 6507 (HUEFS).

Chrysophyllum gonocarpum (Mart. \& Eichler ex Miq.) Engl. (Figura 1c) Grãos de pólen em mônades, médios, isopolares, prolatos, amb. subquadrangular; 4-colporados, planaperturados, ectoaberturas estreitas, endoaberturas lalongadas, curtas e levemente costadas. Exina psilada e muito fina.

Material examinado - BRASIL, Bahia: Ilhéus, Hage 2157 (HUEFS). 
Tabela 1. Caracteres morfométricos dos grãos de pólen das espécies de Chrysophyllum L. (Sapotaceae) ocorrentes na Bahia, Brasil. DP: Diâmetro polar; DE: Diâmetro equatorial; Ex: Exina; Sex: Sexina; Nex: Nexina; FV: Faixa de variação. Medidas em $\mu \mathrm{m}$, índices em números absolutos.

\begin{tabular}{|c|c|c|c|c|c|c|c|}
\hline \multirow{2}{*}{ Espécies/espécimes } & \multicolumn{2}{|l|}{ DP } & \multicolumn{2}{|l|}{$\mathrm{DE}$} & \multirow[b]{2}{*}{ Ex } & \multirow[b]{2}{*}{ Sex } & \multirow[b]{2}{*}{ Nex } \\
\hline & $\bar{x} \pm \mathrm{S} \bar{x}$ & FV & $\overline{\boldsymbol{x}} \pm \mathrm{S} \overline{\boldsymbol{x}}$ & FV & & & \\
\hline \multicolumn{8}{|c|}{ Chrysophyllum arenarium } \\
\hline Lorenzi 6475 & $20,3 \pm 0,2$ & $17,5-22,5$ & $13,9 \pm 0,3$ & $15,0-12,2$ & 1,0 & 0,5 & 0,5 \\
\hline \multicolumn{8}{|c|}{ Chrysophyllum flexuosum } \\
\hline Miranda et al. 6507 & $39,2 \pm 0,5$ & $37,5-42,5$ & $30,4 \pm 0,4$ & $27,5-32,5$ & 2,4 & 0,9 & 1,5 \\
\hline Sztutman 135 & $30,0 \pm 0,5$ & $27,5-35,0$ & $23,6 \pm 0,4$ & $20,0-27,5$ & 2,4 & 0,9 & 1,5 \\
\hline \multicolumn{8}{|c|}{ Chrysophyllum gonocarpum } \\
\hline Hage 2157 & $24,5 \pm 0,4$ & $22,5-27,5$ & $15,3 \pm 0,2$ & $12,5-17,5$ & 1,0 & 0,5 & 0,5 \\
\hline \multicolumn{8}{|c|}{ Chrysophyllum inornatum } \\
\hline Almeida 09 & $30,4 \pm 0,4$ & $27,5-32,5$ & $16,3 \pm 0,3$ & $15,0-17,5$ & 1,6 & 1,1 & 0,5 \\
\hline Barros 2001 & $35,6 \pm 0,4$ & $32,5-40,0$ & $17,3 \pm 0,3$ & $15,0-20,0$ & 1,5 & 0,9 & 0,6 \\
\hline Leite 78 & $32,7 \pm 0,3$ & $30,0-35,0$ & $15,7 \pm 0,2$ & $15,0-17,5$ & 2,4 & 1,5 & 0,9 \\
\hline \multicolumn{8}{|c|}{ Chrysophyllum januariense } \\
\hline Popovkin 1484 & $29,9 \pm 0,3$ & $27,5-32,5$ & $20,0 \pm 0,3$ & $17,5-25,5$ & 1,0 & 0,5 & 0,5 \\
\hline Popovkin 1499 & $37,1 \pm 0,7$ & $35,0-40,0$ & $22,9 \pm 0,2$ & $22,5-25,0$ & 1,0 & 0,5 & 0,5 \\
\hline \multicolumn{8}{|c|}{ Chrysophyllum marginatum } \\
\hline Cielo-Filho 679 & $27,8 \pm 0,3$ & $25,0-30,0$ & $15,3 \pm 0,2$ & $12,5-17,5$ & 2,0 & 1,5 & 0,5 \\
\hline Ferreira 16973 & $24,9 \pm 0,3$ & $22,5-27,5$ & $15,0 \pm 0,5$ & $12,5-17,5$ & 2,0 & 1,5 & 0,5 \\
\hline Santos 812 & $23,8 \pm 0,3$ & $22,5-25,0$ & $15,1 \pm 0,2$ & $15,0-17,5$ & 2,0 & 1,5 & 0,5 \\
\hline \multicolumn{8}{|l|}{ Chrysophyllum rufum } \\
\hline Oliveira 2527 & $25,4 \pm 0,2$ & $22,5-27,5$ & $14,9 \pm 0,3$ & $12,5-17,5$ & 1,6 & 1,1 & 0,5 \\
\hline Queiroz 12048 & $24,8 \pm 0,3$ & $22,5-27,5$ & $16,4 \pm 0,3$ & $12,5-15,0$ & 1,6 & 1,1 & 0,5 \\
\hline Souza 175 & $26,1 \pm 0,3$ & $22,5-30,0$ & $16,0 \pm 0,3$ & $12,5-20,0$ & 1,6 & 1,1 & 0,5 \\
\hline \multicolumn{8}{|c|}{ Chrysophyllum splendens } \\
\hline Marquete 2070 & $27,2 \pm 0,4$ & $25,0-30,0$ & $20,2 \pm 0,3$ & $17,5-22,5$ & 0,5 & - & - \\
\hline Sambuichi 567 & $30,4 \pm 0,4$ & $25,0-32,5$ & $20,3 \pm 0,3$ & $17,5-22,5$ & 0,6 & - & - \\
\hline \multicolumn{8}{|l|}{ Chrysophyllum viride } \\
\hline Ferretti 22 & $28,6 \pm 0,3$ & $27,5-32,5$ & $19,6 \pm 0,4$ & $15,0-22,5$ & 1,0 & 0,5 & 0,5 \\
\hline
\end{tabular}


Figura 1. Grãos de pólen de Chrysophyllum spp. (Sapotaceae) da Bahia. a: C. arenarium - vista equatorial (sup); b: C. flexuosum - vista equatorial (c/op); c: C. gonocarpum - vista polar (c/op); d-f: C. inornatum - d - vista polar (c/op), e - vista equatorial (c/op), f - vista equatorial (sup); g: $C$. januariense - vista equatorial (c/op); h-i: C. marginatum - h - vista equatorial (c/op), $\mathrm{i}-$ vista equatorial (sup); $\mathrm{j}-\mathrm{k}$ : C. rufum $-\mathrm{j}-$ vista equatorial (c/op), $\mathrm{k}-$ vista equatorial (sup); $1-\mathrm{m}$ : C. splendens - 1 - vista equatorial (c/op), $\mathrm{m}-$ vista equatorial (sup); $\mathrm{n}-\mathrm{o}:$ C. viride $-\mathrm{n}-$ vista equatorial (c/op), o - vista equatorial (sup). Barra $=10 \mu \mathrm{m} ; \mathrm{Legenda:} \mathrm{c} / \mathrm{op}=\mathrm{corte}$ óptico, sup = superfície. Fonte: os autores.

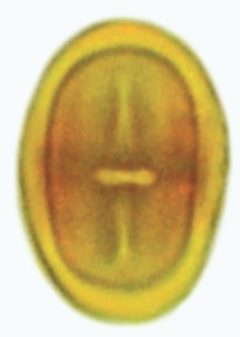

a

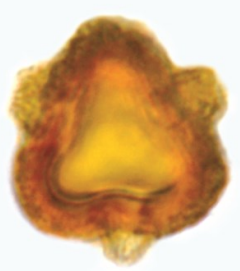

d

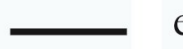

e
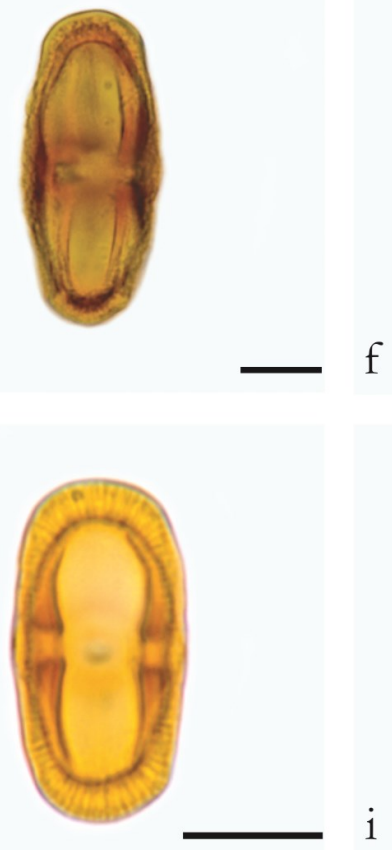

$\mathrm{k}$
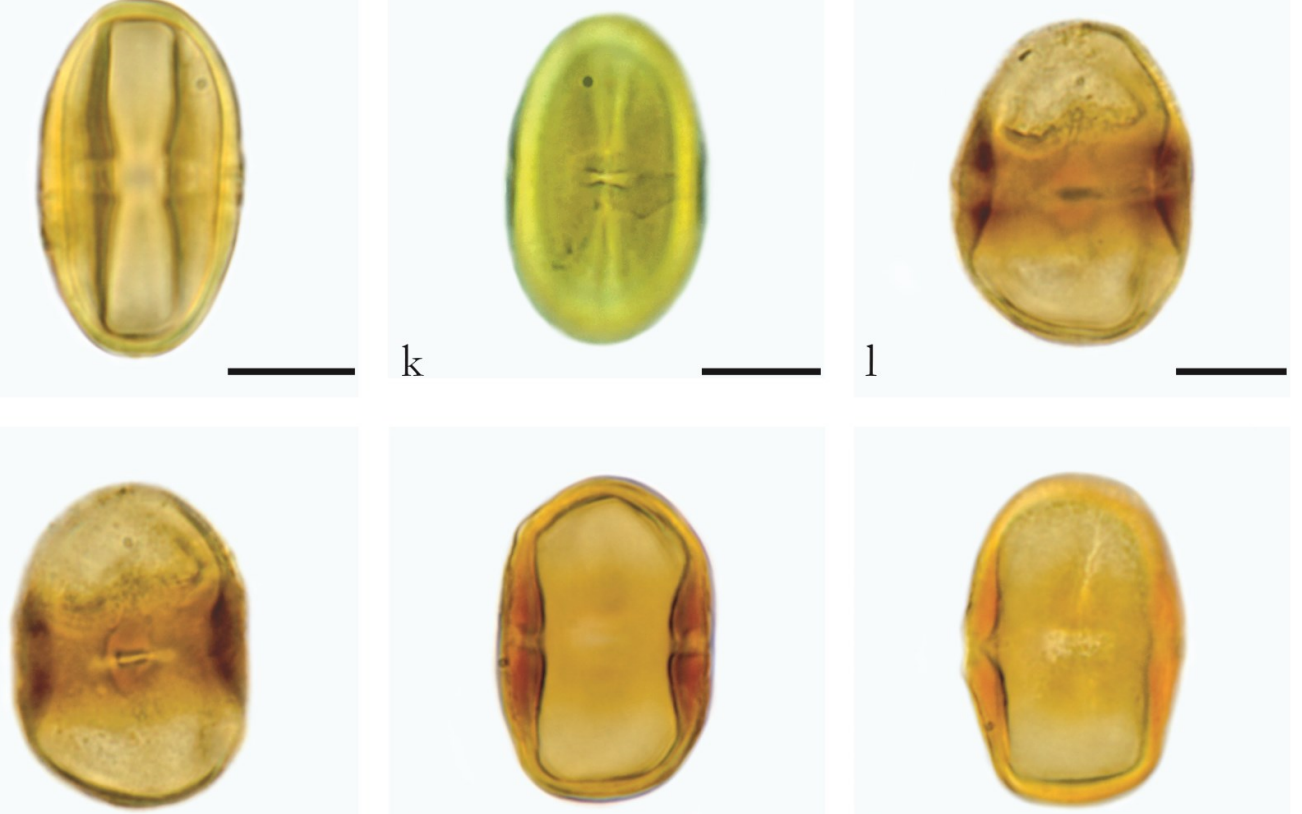

$\mathrm{m}$
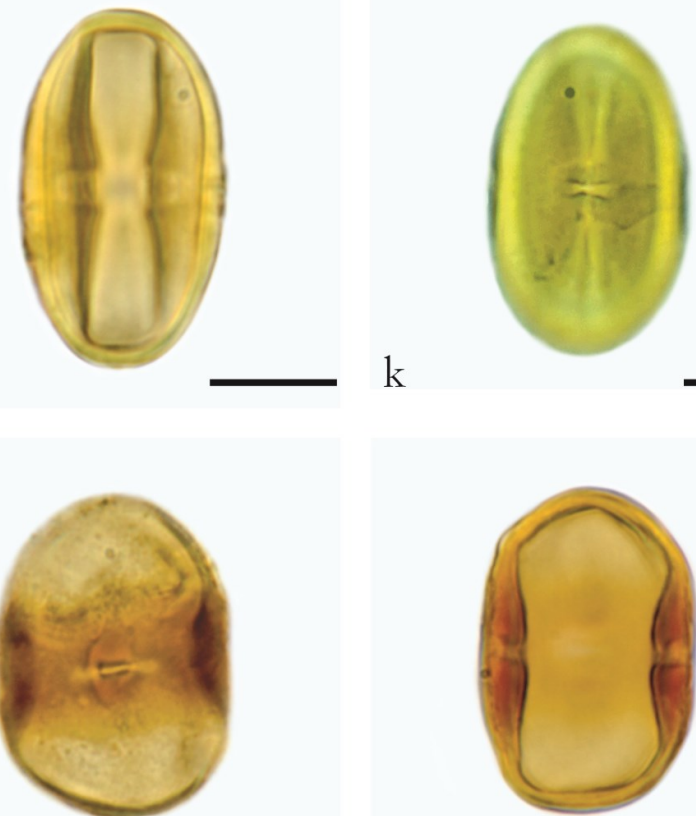

11
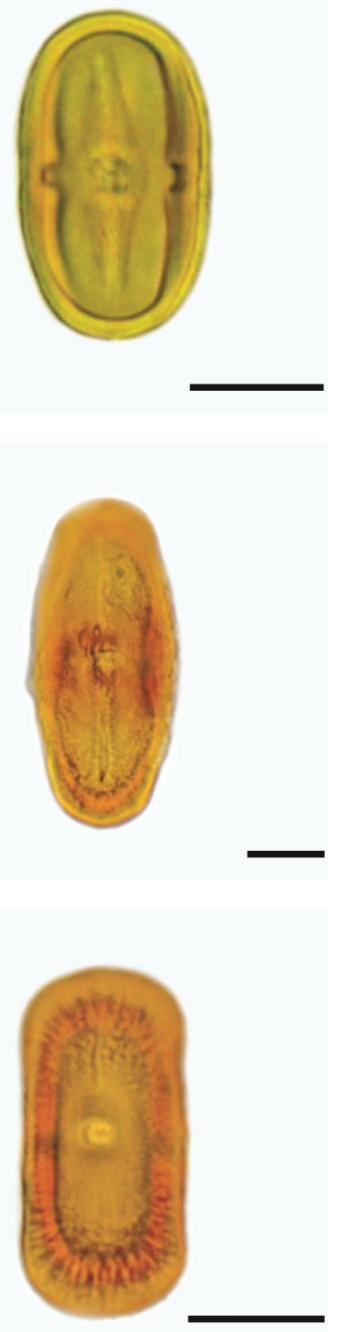

f

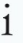

C

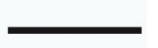

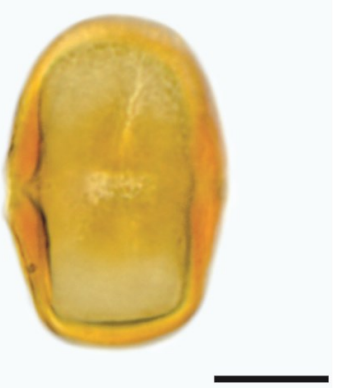


Chrysophyllum inornatum Mart. (Figura 1d-f)

Grãos de pólen em mônades, médios, prolatos, isopolares, amb. triangular, 3-colporados, planaperturados, ectoaberturas estreitas, endoaberturas lalongadas curtas, levemente costadas. Exina microrreticulada, levemente estriada.

Material examinado - BRASIL, São Paulo: Cananéia, 03/ XII/1990, Barros 2001 (HUEFS). Bahia: Uruçuca, 27/V/2000, Leite 78 (HUEFS); Una, 01/XII/2017, Almeida 09 (HUEFS).

\section{Chrysophyllum januariense Eichler (Figura 1g)}

Grãos de pólen em mônades, médios, prolatos, amb. subcircular ou subquadrangular, 3-(4)-colporados, ectoaberturas apresentando margens levemente onduladas, endoaberturas lalongadas, levemente costadas, fastigiadas. Exina psilada.

Material examinado - BRASIL, Bahia: Algodão, Entre Rios, 10/VII/2013, Popovkin 1484 (HUEFS); 31/VII/2013, Popovkin 1499 (HUEFS).

Chrysophyllum marginatum (Hook. \& Arn.) Radlk. (Figura 1h-i) Grãos de pólen em mônades, médios, prolatos, levemente achatado nos pólos, adquirindo um contorno levemente retangular, na vista equatorial, amb. subquadrangular, 4-colporados, ectoaberturas estreitas, endoaberturas lalongadas curtas, levemente costadas. Exina microrreticulada.

Material examinado - BRASIL, São Paulo: Itapeva, 26/ III/2008, Cielo-Filho 679 (HUEFS); Campinas, 05/II/1985, Ferreira 16973 (HUEFS). Bahia: Itaimbé, Santos 812 (HUEFS).

\section{Chrysophyllum rufum Mart. (Figura 1j-k)}

Grãos de pólen em mônades, médios, prolatos, amb. subcircular a subtriangular; 3-colporados, ectoaberturas longas e estreitas, endoaberturas lalongadas curtas, levemente costadas. Exina psilada.

Material examinado - BRASIL, Bahia: Rui Barbosa, 17/I/2006, Queiroz 12048 (HUEFS); Jeremoabo, 15/IX/2006, Oliveira 2527 (HUEFS); Maracás, 23/IV/2002, Souza 175 (HUEFS).

Chrysophyllum splendens Spreng. (Figura 11-m)

Grãos de pólen em mônades, médios, prolatos, amb. subcircular; 3 -(4)-colporados, ectoaberturas de difícil visualização, endoaberturas lalongadas curtas, costadas e presença de fastígio. Exina finamente microrreticulada.

Material examinado - BRASIL, Bahia: Conde, 25/X/1994, Marquete 2070 (HUEFS); Una, 22/V/2005, Sambuichi 567 (HUEFS).

\section{Chrysophyllum viride Mart. \& Eichler (Figura 1n-o)}

Grãos de pólen em mônades, médios, prolatos, amb. subcircular, 4colporados, ectoaberturas estreitas, com margens irregulares, endoaberturas lalongadas, levemente costadas. Exina psiladaà levemente rugulada.

Material examinado - BRASIL, São Paulo: Cunha, 12/II/1996, Ferretti 22 (HUEFS).

\section{Discussão}

Poucos são os estudos realizados sobre a morfologia polínica do gênero Chrysophyllum. O estudo mais abrangente foi desenvolvido por Harley (1991), com as espécies neotropicais de Sapotaceae, no qual é apresentada a descrição dos grãos de pólen para 398 espécies, incluindo algumas espécies de Chrysophyllum.
Os dados aqui apresentados corroboram os poucos dados encontrados na literatura no que se refere à morfologia polínica para espécies de Chrysophyllum, sendo seus grãos de pólen em mônades, médios, isopolares, prolatos, com o tipo apertural 3-4-zonocolporado (Harley, 1991; Perveen; Qaiser, 2002; Melo et al., 2017).

No que se refere ao valor médio dos diâmetros, os grãos de pólen variaram pouco na classe de tamanho, sendo predominante o tamanho médio para todas as espécies analisadas.

Em relação a forma dos grãos de pólen, observou-se o predomínio da forma prolata dentre as espécies estudadas, à exceção da espécie Chrysophyllum flexuosum, caracterizada pelos grãos de pólen subprolatos. Similarmente, Lieux (1980) descreveu para a família Sapotaceae grãos de pólen prolatos, apresentando polos arredondados.

Em relação ao número de aberturas dos grãos de pólen, as espécies estudadas apresentaram variação entre 3 e 4 aberturas, sendo verificado espécies com apenas 3 ou 4 aberturas, enquanto em outras verificou-se a presença de 3 e 4 aberturas. Além do número de aberturas aqui encontrado, Harley (1991) descreveu para a família Sapotaceae espécies com 5 e 6 cólporos. Perveen e Qaiser (2002) também registraram grãos de pólen 5-colporados para Sapotaceae.

Em Chrysophyllum splendens verificou-se a presença de fastígio, no entanto, ressaltamos que, comparando com outros estudos encontramos outras terminologias para o que consideramos fastígio, conforme Punt et al. (2007). Harley (1991) observou que o teto na região da endoabertura é saliente ou protuberante. Da mesma forma, Roubik e Moreno (1991) descreveram a endoabertura protuberante para os grãos de pólen de Pouteria, outro gênero de Sapotaceae. Assim, no presente estudo adotamos o termo fastígio que, possivelmente, representa a mesma característica observada pelos autores citados.

Nos grãos de pólen de Chrysophyllum inornatum, C. marginatum e C. rufum observou-se uma característica diferenciada das demais espécies estudadas quanto à espessura da sexina e nexina. Nessas espécies a sexina apresentou-se mais espessa que a nexina na região polar, característica que também foi observada por Harley (1986) em C. marginatum, com um considerável aumento no comprimento da columela ao redor dos polos, tornando as paredes mais espessas na região polar em relação a região equatorial. Similarmente, Pennington (1991) também observou as mesmas características para $C$. inornatum, C. marginatum e C. subspinosum. Para as demais espécies estudadas verificou-se que nexina e sexina apresentaram espessuras equivalentes na região polar.

A espécie Chrysophyllum marginatum apresentou grãos de pólen com algumas características que diferiram do que foi observado por outros autores. Quanto ao número apertural, foi observado grãos de pólen exclusivamente 4-colporados. No entanto, Melhem et al. (2003) descreveram grãos de pólen apenas 3-colporado. Já em outro estudo, Melo et al. (2017) registraram uma variação no número apertural, ou seja, grãos de pólen 3-(4)-colporados para a mesma espécie. Em relação à ornamentação da exina os grãos de pólen analisados apresentaram -se microrreticulados. Tal característica também foi observada por Melo et al. (2017) com algumas variações no padrão escultural, que se mostrou microrreticulada-rugulada na região equatorial e rugulada-estriada em direção aos pólos. 
Uma característica que se destacou nos grãos de pólen de $C$. flexuosum, C. splendens e C. viride foi a diferença na transparência da parede dos grãos de pólen na região do mesocolpo, sendo esta mais escura em relação ao apocolpo. Essa característica pode estar relacionada ao fato da nexina tornar-se gradualmente mais espessa nessa região, em direção à endoabertura. Por outro lado, já em direção aos polos a coloração se torna mais clara, indicando parede mais delgada naquele sentido. Tais observações não estão associadas à presença de costa na endoabertura, pois conforme Punt et al. (2007), a costa é definida como um espessamento da nexina/ endexina nas margens da endoabertura, ou a ectoabertura.

\section{Conclusão}

Os grãos de pólen de Chrysophyllum apresentaram morfologia polínica com sutis variações, caracterizando este gênero como estenopolínico, mesmo com as pequenas diferenças na ornamentação da exina e variação no número de aberturas. O número de aberturas e a ornamentação da exina são caracteres significativos para a distinção das espécies. Entretanto, nas espécies estudadas, tais caracteres apresentaram-se limitados para a utilização na taxonomia do grupo, o que motiva a continuidade e aprofundamento nas análises em microscopia eletrônica de varredura e transmissão, para a obtenção de melhores resoluções morfopolínicas para o gênero.

Dados da literatura referem que o estado da Bahia é um dos estados mais ricos em diversidade de espécies de Chrysophyllum devido à presença de muitos remanescentes de florestas tropicais úmidas. Nesse contexto, o presente trabalho colabora para o avanço do conhecimento sobre Sapotaceae no Nordeste do Brasil. Ao ser um dos primeiros estudos na Bahia abordando a morfologia polínica do gênero, poderá subsidiar futuros trabalhos tanto local como regional, nas diversas áreas da palinologia e taxonomia do grupo.

\section{Agradecimentos}

Os autores agradecem ao curador do herbário HUEFS, pela disponibilização das amostras; ao Laboratório de Micromorfologia Vegetal (LAMIV) da Universidade Estadual de Feira de Santana, pelo uso das suas instalações e suporte técnico; e ao Conselho Nacional de Desenvolvimento Científico e Tecnológico (CNPq), pela bolsa de iniciação científica concedida à primeira autora.

\section{Financiamento}

O Conselho Nacional de Desenvolvimento Científico e Tecnológico (CNPq) concedeu bolsa de iniciação científica à primeira autora, via cota institucional.

\section{Contribuições de Autoria}

Conceitualização: MACSSS, PPO, CEC. Curadoria de dados: MACSSS, PPO, CEC. Análise formal: MACSSS, PPO, CEC. Aquisição de financiamento: CEC. Investigação: MACSSS. Metodologia: MACSSS, PPO. Administração do projeto: CEC. Recursos: CEC. Programas: MACSSS, PPO. Supervisão: PPO, CEC. Validação: MACSSS, PPO, CEC. Visualização: MACSSS, PPO, CEC. Redação - rascunho original: MACSSS. Redação - revisão e edição: PPO, CEC.

\section{Conflito de Interesse}

Os autores declaram não haver conflitos de interesse a informar.

\section{Disponibilidade dos Dados}

Os dados integrais analisados durante o estudo atual estão apresentados no corpo do manuscrito.

\section{Conformidade ética}

Não se aplica.

\section{Referências}

Alves-Araújo A, Carneiro CE, Faria AD, Terra-Araújo, MH [Internet]. Sapotaceae in Flora do Brasil 2020. [acesso em 18 jul 2020]. Disponível em: http://floradobrasil.jbri.gov.br/reflora/ floradobrasil/FB217

APG IV. An update of the Angiosperm Phylogeny Group classification for the orders and families of flowering plants: APG IV. Botanical Journal of the Linnean Society 2016; 181(1):1-20. doi: $10.1111 /$ boj. 12385

Erdtman G. The acetolysis method. A revised description. Svensk Botanisk Tidskrift 1960; 54:561-564.

Faria AD, Pirani JR, Ribeiro JELS, Nylinder S, Terra-Araújo MH, Vieira PP, Swenson U. Towards a natural classification of Sapotaceae subfamily Chrysophylloideae in the Neotropics. Botanical Journal of the Linnean Society 2017; 185:27-55. doi:10.1093/ botlinnean/box 042

Harley MM. Distinguishing pollen characters for the Sapotaceae. Canadian Journal of Botany 1986; 64:3091-3100. doi:10.1139/ b86-408

Harley MM. The pollen morphology of the Sapotaceae. Kew Bulletin 1991; 46(3):379-491. doi:10.2307/4110538

Judd WS, Campbell CS, Kellogg GEA, Stevens PF, Donoghue MJ. Sistemática vegetal: um enfoque filogenético. Porto Alegre: Artmed; 2009.

Lieux MH. An atlas of pollen of trees, shrubs, and wood vines of Louisiana and other southeastern states, part IV. Sapotaceae to Fabaceae. Pollen et Spores 1980; 24(3-4): 331-368.

Melhem TS, Cruz-Barros MAV, Corrêa AMS, Makino-Watanabe H, Silvestre-Capelato MSF, Esteves VLG. Variabilidade polínica em plantas de Campos do Jordão (São Paulo, Brasil). Boletim do Instituto de Botânica 2003; 16:9-104.

Melo MRF, Corrêa MAS, Cruz-Barros MAV. Flora Polínica da Reserva do Parque Estadual das Fontes do Ipiranga (São Paulo, Brasil). Família 68: Sapotaceae. Hoehnea 2017; 44(1):96-102. doi:10.1590/2236-8906-80/2016

Pennington TD. Flora Neotropica - Monograph 52: Sapotaceae. New York: New York Botanical Garden; 1990.

Pennington TD. Sapotaceae (Sapodilla family). In: Smith N, Mori SA, Henderson A, Stevenson DW, Heald SV. Flowering plants of the Neotropics. New York: The New York Botanical Garden; 2004. p.342-344.

Pennington TD. The genera of Sapotaceae. London: Royal Botanic Gardens; 1991.

Perveen A, Qaiser M. Pollen flora of Pakistan - XXXIV. Sapotaceae. Pakistan Journal of Botany 2002; 34:225-228.

Punt W, Hoen PP, Blackmore S, Nilsson S, Le Thomas A. Glossary of pollen and spore terminology. Review of Palaeobotany and 
Palynology 2007;143(12):1-81. doi:10.1016/

j.revpalbo.2006.06.008

Roubik DW, Moreno PJE. Pollen and spores of Barro Colorado Island. Monographs in Systematic Botany 1991; 36:1-268.

Swenson U, Anderberg AA. Phylogeny, character evolution, and classification of Sapotaceae (Ericales). Cladistics 2005; 21:101130. doi:10.1111/j.1096-0031.2005.00056.x

\section{Como citar este artigo}

How to cite this article

\section{(ABNT)}

SOUZA, M. A. C. S. S.; OLIVEIRA, P. P.; CARNEIRO, C. E.

Morfologia polínica de espécies de Chrysophyllum L. (Sapotaceae) do Estado da Bahia, Brasil. Paubrasilia, Porto Seguro, v. 4, e0066, 2021. DOI 10.33447/paubrasilia.2021.e0066

\section{(Vancouver)}

Souza MACSS, Oliveira PP, Carneiro CE. Morfologia polínica de espécies de Chrysophyllum L. (Sapotaceae) do Estado da Bahia, Brasil. Paubrasilia 2021;4:e0066. doi:10.33447/paubrasilia.2021.e0066 